\title{
Twist-2 matching of transverse momentum dependent distributions at next-to-next-to-leading-order in QCD
}

\section{Daniel Gutierrez-Reyes*†}

Departamento de Física Teórica, Universidad Complutense de Madrid (UCM), E-28040 Madrid, Spain

E-mail: dangut01@ucm.es

\section{Ignazio Scimemi}

Departamento de Física Teórica, Universidad Complutense de Madrid (UCM), E-28040 Madrid, Spain

E-mail: ignaziosefis.ucm.es

\section{Alexey Vladimirov}

Institut für Theorische Physik, Universität Regensburg, D-93040, Regensburg, Germany

E-mail: alexey.vladimirov@ur.de

\begin{abstract}
The factorization theorem for Drell-Yan process and semi inclusive deep inelastic scattering holds for all leading twist transverse momentum distributions. In this context, a QCD perturbative calculation shows several important characteristics of spin-dependent distributions. We consider all the diferent spin-dependent distributions which can be matched onto integrated twist-2 functions, focusing on the transversity and pretzelosity distributions. The pretzelosity case is specially relevant because, using a direct perturbative calculation, we obtain a null result up to two loops which agrees with the experimental measurements. We show the complete set results of the matching at next-to-leading-order and the results focusing on transversity and pretzelosity at next-to-next-toleading-order.
\end{abstract}

23rd International Spin Physics Symposium - SPIN2018 -

10-14 September, 2018

Ferrara, Italy

\footnotetext{
* Speaker.

${ }^{\dagger}$ Proceeding corresponding to the talk given in the Parallel Session: Structure of the Nucleon: TMDs
} 


\section{Introduction}

The recent advances in the study of the transverse momentum dependent distributions (TMD) allow to unravel the structure of hadrons in great detail. Through the so called factorization theorems we construct expressions for cross sections of interesting processes as Drell-Yan or semiinclusive-deep-inelastic-scattering (SIDIS) [1,2,3] in terms of TMD parton distribution functions (TMDPDF) and fragmentation functions (TMDFF). Higher order calculations in QCD for these TMD distributions are important to increase the predictive power of the framework $[4,5]$ in the description of the available experimental data. Increasing the perturbative order in the calculations of TMD distributions allows better theoretical uncertainties.

The efforts to increase the perturbative order of the elements of TMD factorization theorems at next-to-next-to-leading-order (NNLO) have given us the evolution of the TMD distributions up to two and three loops $[6,7,8]$. The unpolarized TMD distribution matchings of TMDPDFs and TMDFFs have been studied up to two loops respectively in $[9,10,11]$ and in [11].

The status of the polarized distributions is less advanced. We focus on the two transvesely polarized distributions: transversity and pretzelosity TMDs. Their matching up to two loops is evaluated in [12]. These two distributions are very interesting because they have been recently subjet of experimental, phenomenological and theoretical investigations. The relevant data for these extractions come mainly from HERMES [13] and COMPASS [14].

The TMD transversity distribution has been extracted using SIDIS data in e.g. [15] with Gaussian models without taking into account the TMD evolution. In these cases the size of the theoretical errors is difficult to estimate. In order to provide this information we need to introduce higher order perturbative information as the calculation of the matching coefficients we are going to recall in this proceeding. For the unpolarized TMD distribution this analysis has been recently done in [5] decreasing the size of the theoretical uncertainties substantially. In principle a similar analysis can be done also for the polarized distribution that we have studied.

For the pretzelosity distribution we outline here the recent analysis made in [16, 17]. In these analyses a practically null value for this distribution is obtained that agrees with our analysis done at up to two loops in $[18,12]$.

\section{Transversely polarized distributions}

The transversity and pretzelosity TMD distributions are derived from a general transversely polarized TMD distribution,

$$
\begin{gathered}
\Phi_{q \leftarrow h}^{\left[i \sigma^{\alpha+} \gamma^{5}\right]}(x, b)= \\
\frac{1}{2} \int \frac{d \lambda}{2 \pi} e^{-i x p^{+} \lambda}\left\langle P, S\left|\bar{T}\left\{\bar{q}(\lambda n+b) \tilde{W}_{n}^{T}(\lambda n+b)\right\} i \sigma^{\alpha+} \gamma^{5} T\left\{\tilde{W}_{n}^{T \dagger}(0) q(0)\right\}\right| P, S\right\rangle,
\end{gathered}
$$

where the index $\alpha$ is transverse and $n$ is a light-like vector. We use the standard notation for the light-cone components of a general vector $v^{\mu}=n^{\mu} v^{-}+\bar{n}^{\mu} v^{+}+g_{T}^{\mu v} v_{v}\left(\right.$ with $\left.n^{2}=\bar{n}^{2}=0, n \cdot \bar{n}=1\right)$. The collinear Wilson lines $W_{n}^{T}(x)$ are rooted at the position $x$ and continued to the infinity along the direction $n$. Its staple contour results in the rapidity divergences, unique feature of TMDs. They are renormalized by the proper rapidity renormalization factor $R$, which is built from the TMD soft 
factor $[19,1,3,6]$

$$
S(b)=\frac{T r_{c o l o r}}{N_{c}}\left\langle 0\left|\left[S_{n}^{T \dagger} \tilde{S}_{\bar{n}}^{T}\right](b)\left[\tilde{S}_{\bar{n}}^{T \dagger} S_{n}^{T}\right](0)\right| 0\right\rangle,
$$

where $S_{n}^{T}$ stands for soft Wilson lines (for a precise definition of collinear and soft Wilson lines see e.g. [11]). In the regularization scheme for rapidity divergences that we use (modified $\delta$ regularization) the $R$ factor can be written easily as $R=S^{-1 / 2}$. This fact makes possible to write a renormalized TMD distribution in a simple way

$$
\Phi^{r e n}(x, b ; \mu, \zeta)=Z(\mu, \zeta \mid \varepsilon) R(b, \mu, \zeta \mid \varepsilon, \delta) \Phi^{u n s u b .}(x, b \mid \varepsilon, \delta)
$$

where $Z$ renormalizes the UV divergences and $R$ the rapidity ones.

The transversely polarized TMD distribution is parameterized in terms of four TMDPDFs. In position space it stands as

$$
\begin{aligned}
\Phi_{q \leftarrow h}^{\left[i \sigma^{\alpha+} \gamma_{5}\right]}(x, b)= & s_{T}^{\alpha} h_{1}(x, b)-i \lambda b^{\alpha} M h_{1 L}^{\perp}(x, b) \\
& +i \varepsilon_{T}^{\alpha \mu} b_{\mu} M h_{1}^{\perp}(x, b)+\frac{M^{2} b^{2}}{2}\left(\frac{g_{T}^{\alpha \mu}}{2}+\frac{b^{\alpha} b^{\mu}}{b^{2}}\right) s_{T \mu} h_{1 T}^{\perp}(x, b),
\end{aligned}
$$

where $s_{T}$ is the transverse part of the hadron spin, $\lambda$ the helicity, $M$ a hadron mass scale and $b$ is the transverse position, Fourier conjugated quantity of the transverse momentum, $q_{T}$. To study the leading twist matching of these distributions we use the operator product expansion (OPE) that allows the expansion of the TMD operator in powers of $b$. The evaluation of the matrix elements of the small- $b$ OPE for our TMD operator results in the following expression

$$
\begin{aligned}
\Phi_{q \leftarrow h}^{\left[i \sigma^{\alpha+} \gamma^{5}\right]}(x, b)=\sum_{f}\left[C_{q \leftarrow f ; t w-2}^{\alpha \beta}(b) \otimes h_{f \leftarrow h}^{\beta ; t w-2}\right](x) & \\
& +b_{\beta} \sum_{f}\left[C_{q \leftarrow f ; t w-3}^{\alpha \beta \gamma}(b) \otimes h_{f \leftarrow h}^{\gamma ; t w-3}\right](x)+\ldots,
\end{aligned}
$$

where $h$ are collinear distributions, $C$ are matching coefficients functions and $\otimes$ symbol stands for the Mellin convolution in the momentum fractions. Extracting particular tensors one can find the matching of individual TMDPDFs onto collinear functions. In particular the tensor structures of transversity $\left(h_{1}\right)$ and pretzelosity $\left(h_{1 T}^{\perp}\right)$ appears in the twist-2 matching, and the other distribution can be produced only at twist-3 [20,21]. The only PDF that contribute to the matching of the twist-2 distributions is the collinear transversity distribution.

The twist- 2 coefficient functions have structures $\sim g_{T}^{\alpha \beta}$ and $\sim b^{\alpha} b^{\beta} / b^{2}$. So, the natural decomposition of this function is

$$
C_{q \leftarrow f ; t w-2}^{\alpha \beta}(x, b)=g_{T}^{\alpha \beta} \delta C_{q \leftarrow f}\left(x, \mathbf{L}_{\mu}\right)+\left(\frac{g_{T}^{\alpha \beta}}{2(1-\varepsilon)}+\frac{b^{\alpha} b^{\beta}}{b^{2}}\right) \delta^{\perp} C_{q \leftarrow f}\left(x, \mathbf{L}_{\mu}\right),
$$

where $\varepsilon$ is the parameter of dimensional regularization $(d=4-2 \varepsilon)$ and $\mathbf{L}_{\mu}=\ln \left(\frac{\mu^{2} b^{2}}{4 e^{-2 \gamma_{E}}}\right)$ is the only way in which the matching coefficients depend on the transverse position. As the pieces of 
the decomposition do not mix each other, we find individual matching for each TMD distributions,

$$
\begin{aligned}
h_{1}^{q}(x, b) & =\int_{x}^{1} \frac{d y}{y} \sum_{f=q, \bar{q}} \delta C_{q \leftarrow f}\left(\frac{x}{y}, \mathbf{L}_{\mu}\right) h_{1}^{f}(y)+\mathscr{O}\left(b^{2}\right), \\
h_{1 T}^{\perp, q}(x, b) & =\frac{2}{b^{2} M^{2}} \int_{x}^{1} \frac{d y}{y} \sum_{f=q, \bar{q}} \delta^{\perp} C_{q \leftarrow f}\left(\frac{x}{y}, \mathbf{L}_{\mu}\right) h_{1}^{f}(y)+\mathscr{O}\left(b^{2}\right) .
\end{aligned}
$$

\section{Renormalization group equations and evolution}

The dependence on the renormalization scales of the distributions studied is given by a pair of evolution equations.

$$
\begin{aligned}
& \mu^{2} \frac{d}{d \mu^{2}} \Phi^{r e n}(x, b ; \mu, \zeta)=\frac{\gamma_{F}(\mu, \zeta)}{2} \Phi^{r e n}(x, b ; \mu, \zeta), \\
& \zeta \frac{d}{d \zeta} \Phi^{r e n}(x, b ; \mu, \zeta)=-\mathscr{D}(\mu, b) \Phi^{r e n}(x, b ; \mu, \zeta) .
\end{aligned}
$$

A detailed study of this system of equations is presented in [5]. The anomalous dimensions are defined via the corresponding renormalization constants and their values are know up to three-loop order. They satisfy the integrability condition

$$
\zeta \frac{d}{d \zeta} \frac{\gamma_{F}^{i}(\mu, \zeta)}{2}=\mu^{2} \frac{d}{d \mu^{2}}\left(-\mathscr{D}^{i}(\mu, b)\right)=-\frac{\Gamma^{i}(\mu)}{2}
$$

which guaraties the existence of a common solution for the equations $(3.1,3.2)$. Here $\Gamma^{i}$ is the so called cusp anomalous dimension. In impact parameter space a common solution of these equations is found straightforwardly

$$
\Phi^{r e n}\left(z, b, Q R, \mu_{f}, \zeta_{f}\right)=\exp \left[\int_{P}\left(\gamma_{F}^{i}(\mu, \zeta) \frac{d \mu}{\mu}\right)-\mathscr{D}^{i}(\mu, b) \frac{d \zeta}{\zeta}\right] \Phi^{r e n}\left(z, b, Q R, \mu_{i}, \zeta_{i}\right)
$$

Thus, the so called TMD evolution kernel is defined as

$$
R^{i}\left[b ;\left(\mu_{f}, \zeta_{f}\right) \rightarrow\left(\mu_{i}, \zeta_{i}\right)\right]=\exp \left[\int_{P}\left(\gamma_{F}^{i}(\mu, \zeta) \frac{d \mu}{\mu}\right)-\mathscr{D}^{i}(\mu, b) \frac{d \zeta}{\zeta}\right]
$$

where the integration is performed along a path $\mathrm{P}$ in the $(\mu, \zeta)$-plane. In principle, the integration can be done on an arbitrary path $\mathrm{P}$ and the solution is independent on it, thanks to the integrability condition in Eq. 3.3. But, at a finite perturbation theory order the condition of the Eq. 3.3 is violated by the next perturbative order. As a consecuence, the expression of the evolution factorn in dependent on the chosen integration paths. The restoration of path-independence is studied in [5]. Here we show one of the path independent solutions that is written as

$$
R^{i}\left[b ;\left(\mu_{f}, \zeta_{f}\right) \rightarrow\left(\mu_{i}, \zeta_{i}\right) ; \mu_{0}\right]=\exp \left[\int_{\mu_{i}}^{\mu_{f}} \frac{d \mu}{\mu} \gamma_{F}^{q}\left(\mu, \zeta_{f}\right)-\int_{\mu_{0}}^{\mu_{i}} \frac{d \mu^{\prime}}{\mu^{\prime}} \ln \left(\frac{\zeta_{f}}{\zeta_{i}}\right)\right]\left(\frac{\zeta_{f}}{\zeta_{i}}\right)^{-\mathscr{D}_{\text {Pert }}^{q}\left(\mu_{0}, b\right)-g_{k} b^{2}}
$$

where $\mathscr{D}_{\text {pert }}^{q}$ is the perturbative expression for the rapidity anomalous dimension and $g_{K}$ is a parameter that takes into account the purely non-perturbative part of the evolution factor. In the Fig. 1, the evolution factor for the quark case $(i=q)$ is shown up to NNLO for different energies of current experiments. 


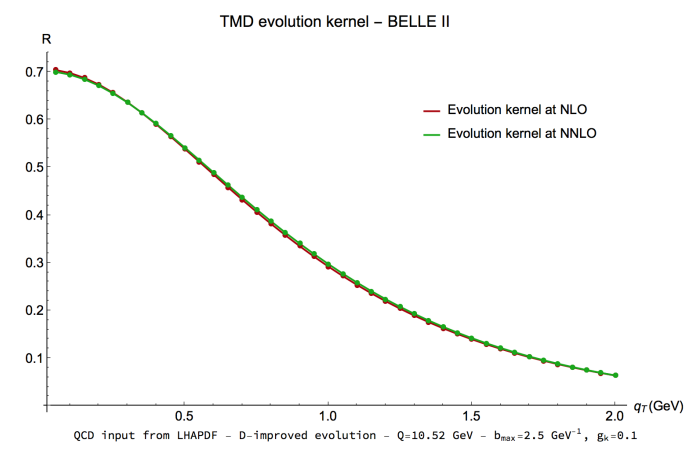

(a) Belle II CM energy

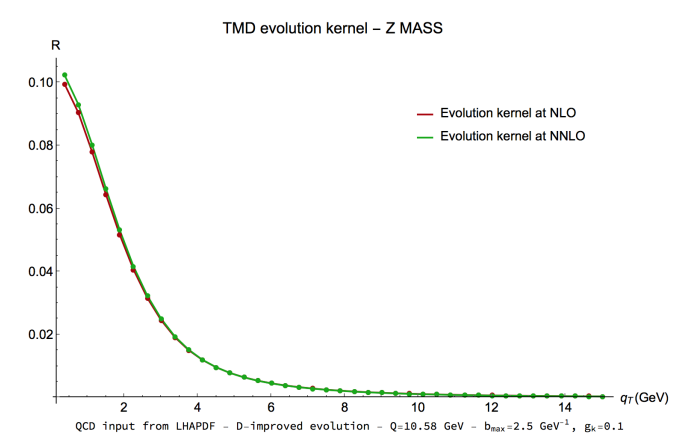

(b) Lep CM energy

Figure 1: Plots of the TMD evolution kernel at next-to-leading-order (NLO) and next-to-next-to-leadingorder (NNLO) for center of mass energies related to Belle II experiment (a) and to Lep experiment (b)

\section{Matching of the transversity distribution at NNLO}

The calculation of the matching coefficients for the transversity distribution is straighforward from the unpolarized one done in [11]. The main difference is the non-mixing with gluons. This calculation stands as an explicit evidence that the factorization theorems of spin dependent TMD distributions work properly. We check explicitly up to NNLO that the UV and rapidity renormalization constants are exactly the same that the ones used for the unpolarized TMD distribution that enters in spin independent factorization theorems. The evolution equations for spin dependent TMD distributions and for the coefficients that result from its matching onto integrated PDFs have the same form that the ones for the unpolarized TMD, see e.g. [12].

In perturbation theory the matching coefficients can be written as

$$
\delta C_{f \leftarrow f^{\prime}}\left(x, \mathbf{L}_{\mu}, \mathbf{l}_{\zeta}\right)=\sum_{n=0}^{\infty} a_{s}^{n} \sum_{k=0}^{n+1} \sum_{l=0}^{n} \mathbf{L}_{\mu}^{k} \mathbf{l}_{\zeta}^{l} \delta C_{f \leftarrow f^{\prime}}^{(n ; k, l)}(x),
$$

where $a_{s}=g^{2} /(4 \pi)^{2}$ and $\mathbf{l}_{\zeta}=\ln \left(\mu^{2} / \zeta\right)$ are the rapidity logarithms. The coefficients $\delta C^{(n ; k, l)}$ with $k+l>0$ are fixed order-by-order with the help of the renormalization group above-named. Thus, the only part that cannot be calculated in this way are the coefficients $\delta C^{(n ; 0,0)}$. We show here the result up to NLO,

$$
\begin{gathered}
\delta C_{f \leftarrow f^{\prime}}^{[0]}=\delta C_{f \leftarrow f^{\prime}}^{(0 ; 0,0)}(x)=\delta_{f f^{\prime}} \delta(1-x), \\
\delta C_{f \leftarrow f^{\prime}}^{(1 ; 0,0)}(x)=-C_{F} \zeta_{2} \delta_{f f^{\prime}} \delta(1-x) .
\end{gathered}
$$

that agrees with the results obtained in $[22,18,23]$.

The new feature for the NNLO calculation is the mixing with the anti-quark operator. The results are quite lengthly and we omit them in this proceeding. To see their explicit expressions see Eqs. $(4.9,4.10)$ (and Eqs. $(6.9,6.10)$ for the fragmentation case) of [12]. One interesting feature of the NNLO transversity matching coefficients is its relation with the unpolarized ones. Both coefficients can be written as

$$
C^{(2 ; 0,0)}(x)=P^{[1]}(x) F_{1}(x)+F_{2}(x)+\delta(1-x) F_{3},
$$


where $P^{[1]}(x)$ is LO DGLAP kernel of the corresponding PDF. The function $F_{1}(x)$ and the constant $F_{3}$ are exactly the same for unpolarized and transversity cases. That behavior is expected because these parts are the ones proportional to $1 /(1-x)$ and $\delta(1-x)$ contributions, respectively. They come from diagrams where the quark interact with the Wilson lines and are insensitive to the polarization structure of the operator. The only different part is the non-singular (at $x \rightarrow 1$ ) function $F_{2}(x)$.

\section{Matching of the pretzelosity distribution at NNLO}

The calculation of the matching of the TMD pretzelosity distribution over the integrated transversity PDF is similar to the one of the transversity TMD distribution. The one-loop result was given in [18],

$$
\delta^{\perp} C_{q \leftarrow q}^{[1]}(x, b)=-4 C_{F} B^{\varepsilon} \Gamma(-\varepsilon) \bar{x} \varepsilon^{2}=0+\mathscr{O}(\varepsilon),
$$

where $\bar{x}=1-x$ and $B=b^{2} / 4$. We see that the result is $\varepsilon$-supressed, as we anticipated before.

To calculate the matching coefficient at NNLO we have to obtain the contribution of the pretzelosity TMDPDF at NNLO and the contribution of the matching of the pretzelosity 1-loop coefficient convoluted with the integrated transversity PDF. It is interesting to organize the result of the sum of the NNLO diagrams by the different color factors that appear,

$$
\delta^{\perp} \Phi_{f \leftarrow f^{\prime}}^{[2]}=C_{F}^{2} A_{F}+C_{F}\left(C_{F}-\frac{C_{A}}{2}\right) A_{F A}+\frac{C_{F} C_{A}}{2} A_{A}+C_{F} N_{f} A_{N},
$$

because can see major cancellations between them, taking into account that $A_{F A}=A_{A}+$ $\mathscr{O}(\varepsilon), A_{N}=\mathscr{O}(\varepsilon)$. So, the renormalized pretzelosity TMDPDF does not depend on $C_{A}$ and can be written as

$$
\begin{gathered}
h_{1 T, q \leftarrow q}^{\perp[2]}=-4 C_{F}^{2}(\bar{x}(3+4 \ln \bar{x})+4 x \ln x)+\mathscr{O}(\varepsilon), \\
h_{1 T, q \leftarrow \bar{q}}^{\perp[2]}=0 .
\end{gathered}
$$

We find that the equation for the matching of the pretzelosity TMD onto the transversity integrated PDF reads as

$$
\begin{gathered}
\delta^{\perp} C_{q \leftarrow q}^{[2]}(x, b)=h_{1 T, q \leftarrow q}^{[2]}(x, b)-\left[\delta^{\perp} C_{q \leftarrow q}^{[1]}(b) \otimes \delta f_{q \leftarrow q}^{[1]}\right](x) \\
\delta^{\perp} C_{q \leftarrow \bar{q}}^{[2]}(x, b)=0 .
\end{gathered}
$$

The result for the convolution term is the same that for the coefficient in (5.3). Thus,

$$
\delta^{\perp} C_{q \leftarrow f}^{[2]}(x, b)=0+\mathscr{O}(\varepsilon)
$$

where $f=q, \bar{q}$. 


\section{Conclusions}

In this article the matching of the two transversely polarized TMD distributions at leading twist is shown. These results give us the first calculation of a polarized TMD (trasversity) at the same level of accuracy of the unpolarized TMD distribution. As is discussed in [4], to increase the perturbative precision in the matching coefficients allows to decrease the theoretical errors and gives us more accurate information of the nonperturbative contributions. The improvement in the theory for polarized distributions shown in this article opens the path to phenomenological analyses with the same level of precision that the obtained for unpolarized TMD.

For another part, this calculation checks explicitly the spin independence of the TMD factorization theorems up to NNLO. Further on, this calculation stands also for a check of the spin independence of the double-scale evolution of the TMD distributions.

For the pretzelosity, an unexpected null result is found up to two-loop level. Some signs to stand that the twist-2 matching of this distribution is zero at all orders in perturbation theory are encountered, But these statements are not a complete demonstration for the nullity of this matching at all orders. We conjecture that the twist- 2 matching of the pretzelosity function is zero at all orders and only the first non-zero matching appears at twist-4 level.

\section{References}

[1] J. Collins, Foundations of perturbative QCD. Cambridge University Press, 2013.

[2] M. G. Echevarria, A. Idilbi and I. Scimemi, Soft and Collinear Factorization and Transverse Momentum Dependent Parton Distribution Functions, Phys. Lett. B726 (2013) 795-801, [1211.1947].

[3] A. Vladimirov, Structure of rapidity divergences in soft factors, JHEP 04 (2018) 045, [1707.07606].

[4] I. Scimemi and A. Vladimirov, Analysis of vector boson production within TMD factorization, Eur. Phys. J. C78 (2018) 89, [1706.01473].

[5] I. Scimemi and A. Vladimirov, Systematic analysis of double-scale evolution, JHEP 08 (2018) 003, [1803.11089].

[6] M. G. Echevarria, I. Scimemi and A. Vladimirov, Universal transverse momentum dependent soft function at NNLO, Phys. Rev. D93 (2016) 054004, [1511.05590].

[7] Y. Li and H. X. Zhu, Bootstrapping Rapidity Anomalous Dimensions for Transverse-Momentum Resummation, Phys. Rev. Lett. 118 (2017) 022004, [1604.014 04 ].

[8] A. A. Vladimirov, Soft-/rapidity- anomalous dimensions correspondence, Phys. Rev. Lett. 118 (2017) 062001, [1610.05791].

[9] S. Catani and M. Grazzini, Higgs Boson Production at Hadron Colliders: Hard-Collinear Coefficients at the NNLO, Eur. Phys. J. C72 (2012) 2013, [1106.4652].

[10] T. Gehrmann, T. Lubbert and L. L. Yang, Transverse parton distribution functions at next-to-next-to-leading order: the quark-to-quark case, Phys. Rev. Lett. 109 (2012) 242003, [1209.0682]. 
[11] M. G. Echevarria, I. Scimemi and A. Vladimirov, Unpolarized Transverse Momentum Dependent Parton Distribution and Fragmentation Functions at next-to-next-to-leading order, JHEP 09 (2016) 004, [1604.07869].

[12] D. Gutierrez-Reyes, I. Scimemi and A. Vladimirov, Transverse momentum dependent transversely polarized distributions at next-to-next-to-leading-order, JHEP 07 (2018) 172, [18 05.07243 ].

[13] HERMES collaboration, A. Airapetian et al., Effects of transversity in deep-inelastic scattering by polarized protons, Phys. Lett. B693 (2010) 11-16, [1006.4221].

[14] COMPASS collaboration, C. Adolph et al., Collins and Sivers asymmetries in muonproduction of pions and kaons off transversely polarised protons, Phys. Lett. B744 (2015) 250-259, [1408 . 4405 ].

[15] M. Anselmino, M. Boglione, U. D’Alesio, S. Melis, F. Murgia and A. Prokudin, Simultaneous extraction of transversity and Collins functions from new SIDIS and e+e-data, Phys. Rev. D87 (2013) 094019, [1303.3822].

[16] C. Lefky and A. Prokudin, Extraction of the distribution function $h_{1 T}^{\perp}$ from experimental data, Phys. Rev. D91 (2015) 034010, [1411.0580].

[17] B. Parsamyan, Measurement of target-polarization dependent azimuthal asymmetries in SIDIS and Drell-Yan processes at COMPASS experiment, PoS QCDEV2017 (2018) 042.

[18] D. Gutierrez-Reyes, I. Scimemi and A. A. Vladimirov, Twist-2 matching of transverse momentum dependent distributions, Phys. Lett. B769 (2017) 84-89, [1702.06558].

[19] M. G. Echevarria, A. Idilbi and I. Scimemi, Factorization Theorem For Drell-Yan At Low $q_{T}$ And Transverse Momentum Distributions On-The-Light-Cone, JHEP 07 (2012) 002, [1111. 4996 ].

[20] I. Scimemi and A. Vladimirov, Matching of transverse momentum dependent distributions at twist-3, Eur. Phys. J. C78 (2018) 802, [1804.08148].

[21] I. Scimemi, A. Tarasov and A. Vladimirov, Collinear matching for Sivers function at next-to-leading order, 1901.04519.

[22] A. Bacchetta and A. Prokudin, Evolution of the helicity and transversity Transverse-Momentum-Dependent parton distributions, Nucl. Phys. B875 (2013) 536-551, [1303.2129].

[23] M. G. A. Buffing, M. Diehl and T. Kasemets, Transverse momentum in double parton scattering: factorisation, evolution and matching, JHEP 01 (2018) 044, [1 708 . 03528]. 